\title{
Prevenção às ISTs/HIV entre mulheres lésbicas e bissexuais: uma revisão bibliográfica (2013-2017)
}

\author{
Prevención de las ETS/VIH entre lesbianas y bisexuales: una revisión bibliográfica \\ (2013-2017)
}

\section{Prevention of STI/HIV between lesbian and bisexual women: a bibliographic research (2013-2017)}

\author{
Amanda Dal Santo \\ Universidade Estadual do Centro-Oeste (Unicentro), Irati - PR/Brasil \\ ORCID: 0000-0001-5261-466X \\ E-mail: dalsantoamanda@gmail.com
}

Gustavo Zambenedetti

Universidade Estadual do Centro-Oeste (Unicentro), Irati - PR/Brasil ORCID: 0000-0002-7372-9930

E-mail: gugazam@yahoo.com.br

\begin{abstract}
Resumo
A prevenção às ISTs/HIV deve considerar os diferentes aspectos que podem colocar determinadas populações em situação de vulnerabilidade. Com base nesta compreensão, esta pesquisa realizou uma revisão bibliográfica acerca da prevenção às ISTs/HIV entre mulheres lésbicas e bissexuais. As plataformas de busca utilizadas foram a Scielo e a Biblioteca Virtual em Saúde (BVS), abrangendo artigos em inglês, português e espanhol, publicados entre 2013 e 2017. Apresentamos os resultados e discussão sob a forma de linhas de análise. Estas revelam aspectos que atuam na vulnerabilidade do público estudado, como a percepção acerca da orientação sexual, raça, estigma, despreparo das/os profissionais de saúde e desconhecimento/conhecimento impreciso com relação a métodos de barreira. Apontamos para a necessidade da criação de políticas que abordem o tema estudado, considerando sua complexidade e a diversidade de vivências a partir do atravessamento de diferentes marcadores sociais. Por fim, indicamos sugestões de novos estudos a serem realizados.
\end{abstract}

Palavras-chaves: Prevenção; IST; HIV; Lésbicas; Bissexuais.

\section{Resumen}

La prevención de ETS/VIH debe considerar los diferentes aspectos que pueden colocar ciertas poblaciones en la situación de vulnerabilidad. En base a esta comprensión, esta investigación llevó a cabo una revisión bibliográfica de la prevención de las ETS/VIH entre lesbianas y bisexuales. Las plataformas de búsqueda utilizadas fueron la Scielo e la Biblioteca Virtual en Salud (BVS), que cubren artículos en inglés, portugués y español, publicados entre 2013 y 2017. Presentamos los resultados y discusiones en forma de lineas de análisis. Estos revelan aspectos que actúan sobre la vulnerabilidad del público, como las percepciones sobre la orientación sexual, el racismo, el estigma, el descuido de los profesionales de la salud y el descontento/conocimiento inexacto de las mujeres relacionadas con los métodos de barrera. Señalamos la necesidad de desarrollar políticas que abordan el emita, considerando su complejidad y diversidad de experiencias de diferentes marcadores sociales. Al final, presentamos sugerencias para que se hagan nuevos estudios en el campo temático.

Palabras clave: Prevención; ETS; VIH; Lesbiana; Bisexual.

\footnotetext{
Abstract

Prevention in STIs/HIV must consider different aspects that can put certain populations in situation of vulnerability. Based on this understanding, this research carried out a bibliographic research about prevention of STIs/HIV among lesbian and bisexual women. The search platforms used were Scielo and the Virtual Health Library (VHL), covering articles in English, Portuguese and Spanish, published between 2013 and 2017. We presented the results and discussion in the form of lines of analysis. These reveal aspects of the vulnerability of the studied public, such as the perception of sexual
} 
orientation, race, stigma, unpreparedness of health professionals and lack of knowledge/imprecise knowledge of women related to barrier methods. We point to the need to create policies that address the theme, considering its complexity and the diversity of experiences from the crossing of different social markers. At the end, we indicate suggestions for new studies to be carried out on this theme.

Keywords: Prevention; STI; HIV; Lesbian; Bisexual.

\section{Introdução}

A necessidade de investigar as produções acadêmicas existentes que abordam o contexto de prevenção às Infecções Sexualmente Transmissíveis (ISTs) e HIV (Vírus da Imunodeficiência Humana) entre mulheres lésbicas e bissexuais foi construída diariamente na vivência da primeira autora deste artigo enquanto mulher lésbica. A necessidade de protagonizar esta pesquisa expressa uma dimensão política, pois surgiu como uma forma de afirmar a existência de mulheres lésbicas e bissexuais, de compor com a construção de materiais que atendam as demandas específicas desses grupos e de auxiliar no movimento de reivindicação do direito ao acesso universal e equitativo à saúde. Junto a isto, há a nossa motivação enquanto pesquisadora e pesquisador do campo da prevenção, que buscam a equidade do acesso à saúde, afirmando uma abordagem que leve em consideração os direitos humanos e os direitos sexuais (Carrara, 2010).

Quando analisamos o contexto dos movimentos de enfrentamento da epidemia da AIDS nos anos 1980, a temática saúde da mulher lésbica e bissexual não aparece com destaque entre as pautas levantadas, e isso pode apontar para o entendimento do porquê ela ainda é tão pouco discutida. Nesse período, diversos movimentos sociais emergiram e se aliaram ao enfrentamento da epidemia, protagonizados predominantemente por homens gays (Ministério da Saúde, 2013). Os grupos exclusivamente de mulheres lésbicas e bissexuais surgiram justamente por conta da necessidade da autoafirmação dessas identidades dentro do movimento LGBTQ+, assim como do movimento feminista e negro (Barbosa, Nascimento, Carvalho \& Cavalcante, 2014). A partir dessas organizações, houve também um aumento do interesse voltado à saúde dessas mulheres e de suas demandas específicas (Facchini \& Barbosa, 2006), mas ainda não foi suficiente para desencadear em ações preventivas voltadas para saúde lésbica e bissexual, pois além de invisibilizadas dentro dos movimentos sociais, elas não eram contempladas nos então chamados "grupos de risco da AIDS” (Ministério da Saúde, 2013).

O apagamento lésbico e bissexual e as forças que limitam essas existências nos espaços sociais são reflexos de sistemas e fenômenos institucionais complexos que estruturam a sociedade, como o patriarcado ${ }^{1} \mathrm{e}$ a heterossexualidade compulsória. Adrienne Rich (2010) aponta esta como uma instituição política que fortalece as opressões sofridas pelas mulheres, diminuindo seu poder. Assim, o modelo heterossexual é visto como uma imposição, reforçada e naturalizada por discursos religiosos, censuras históricas e imagens midiáticas - como a idealização do amor romântico heterossexual - estando a favor do patriarcado e negando a existência de mulheres lésbicas e bissexuais. Por conta disso, o apagamento dessas existências não ocorre somente no campo da saúde, podendo ser visualizado em outros setores, como na educação, na economia e na mídia. Chama-nos a atenção o fato de que o primeiro dossiê de lesbocídio (assassinato de mulheres lésbicas por ódio ou discriminação) (Peres, Soares \& Dias, 2017) - foi divulgado apenas em 2018.

\footnotetext{
${ }^{1}$ Em "Gênero: a história de um conceito" (2009), Adriana Piscitelli define o patriarcado como "um sistema social no qual a diferença sexual serve como base da opressão e da subordinação da mulher pelo homem" (p.132).
} 
Também devemos considerar que mulheres lésbicas e bissexuais não são um grupo homogêneo e possuem experiências distintas. Por conta disso, tomamos a interseccionalidade como perspectiva e categoria de análise nos resultados e discussão. A interseccionalidade compreende que as relações de poder desencadeiam em formas conjugadas de opressão a partir de diferentes marcadores sociais - como raça, gênero, classe e sexualidade - que se potencializam na medida em que se entrecruzam (Hirata, 2014; López, 2011). Nessa perspectiva, esses marcadores devem ser analisados de forma indissociada e não-hierarquizada, pois entende-se que as bases estruturantes da sociedade, como o racismo e o sexismo, coexistem, não sendo possível analisar um sem o outro. Não se trata de uma somatória de marcadores, mas da produção de experiências e corpos distintos a partir desses entrecruzamentos.

Tomamos ainda como instrumento de análise nesta revisão o conceito de vulnerabilidade apresentado por Ayres, Junior, Calazans e Saletti (2009). A vulnerabilidade compreende a suscetibilidade ao adoecimento e às ISTs como advinda de um conjunto de aspectos que para além de individuais, são também sociais, coletivos e contextuais, influenciando na disponibilidade de acesso à recursos de proteção pelos grupos vulnerabilizados. Dessa forma, os marcadores sociais também podem ser vistos como aspectos que produzem relações de vulnerabilidade às ISTs/HIV.

Diante disso, esta pesquisa teve como objetivo analisar a literatura acerca da prevenção de ISTs/HIV entre mulheres lésbicas e bissexuais, contribuindo para o conhecimento do estado da arte, para a compreensão das vulnerabilidades associadas a esse público e também para a indicação de pontos que possam ser ampliados em novas produções. Destacamos a relevância desse estudo para o campo da formulação de políticas públicas, do aperfeiçoamento de abordagens profissionais na saúde no que tange os temas prevenção de ISTs/HIV e diversidade sexual e também como material a ser utilizado pelos movimentos sociais na reinvidicação do direito à saúde de mulheres lésbicas e bissexuais.

\section{Método}

Realizamos uma pesquisa bibliográfica que, segundo Gil (2002), é desenvolvida a partir de materiais já elaborados, permitindo com que a pesquisadora ou o pesquisador entre em contato com um amplo conjunto de fenômenos. Por possuir caráter exploratório, optamos por utilizar a perspectiva da revisão narrativa. De acordo com Rother (2007), esta é de caráter aberto, flexível e qualitativo, possibilitando conhecer o estado da arte de determinado contexto.

A coleta de dados foi realizada através da Scientific Electronic Library Online (SciELO) e do portal da Biblioteca Virtual em Saúde (BVS), selecionado para a amostra da pesquisa apenas artigos, publicados entre 2013 e 2017, em língua inglesa, espanhola e portuguesa. Optamos por abranger essas três linguas com o intuito de ampliar a compreensão acerca do tema investigado em diferentes países. O período delimitado visou a fornecer um panorama atualizado acerca da temática investigada. Iniciamos a busca em outubro de 2018 e finalizamos em janeiro de 2019. Excluímos os artigos duplicados, não disponíveis em seu formato completo ou que não tinham relação com o tema da pesquisa. As palavras-chaves utilizadas na busca foram "STI", "STD" e "HIV" em cruzamento com "lesbian", "bisexual" e "female homosexuality". Apesar da seleção das palavras-chaves, a grande maioria dos materiais encontrados abordavam questões do contexto preventivo de ISTs/HIV de homens que se relacionam sexualmente com homens, sendo tais artigos excluídos. Esta seleção foi possível através da leitura dos resumos dos artigos.

Através dos critérios mencionados, selecionamos 23 artigos, os quais foram lidos por ordem de publicação e analisados. Os resultados e a discussão são apresentados de forma integrada. Inicialmente, mostramos uma caracterização dos estudos. $\mathrm{Na}$ sequência, 
apresentamos linhas de análise que revelam aspectos que atuam na ampliação ou diminuição da vulnerabilidade do público estudado, contribuindo para a discussão da prevenção às ISTs/HIV.

\section{Resultados e Discussão Caracterização dos estudos}

Apesar da amostra do estudo abranger artigos em inglês, português e espanhol, apenas quatro artigos foram feitos e publicados na América Latina (Batista \& Zambenedetti, 2017; Carvalho et al., 2013; Mora \& Monteiro, 2013; Silberman, Buedo \& Burgos, 2016). Também, apenas três artigos da SciELO foram selecionados (Mora \& Monteiro, 2013; Muranda, Mugo, \& Antonites, 2014; Palma \& Orcasita, 2017), sendo as outras vinte fontes da BVS. Há um maior número de artigos publicados em 2013 e 2014, resultando em 6 artigos por ano, enquanto em 2016 e 2017 foram publicados 3 artigos. Há também uma predominância de estudos realizados nos Estados Unidos e Canadá, de cunho quantitativo e transversal. A maioria dos estudos foi realizado com mulheres, predominantemente jovens ou adultas, sendo que apenas dois artigos abrangeram adolescentes (Doull et al., 2017; Muzny, Harbison, Pembleton, \& Austin, 2013) e nenhum incluiu mulheres idosas.

\section{Orientação sexual x Práticas x Desejos}

Há uma variância nos termos utilizados para se referir às mulheres que se relacionam com mulheres dentro desses estudos, sendo possível dividi-los em duas categorias. A primeira é dos estudos que focam predominantemente na orientação sexual, utilizando os termos: lésbicas, bissexuais, minoria sexual feminina, queer e predominantemente lésbica ou bissexual. E a

\footnotetext{
2 Utilizamos o termo "não-heterossexual" para tentar abarcar uma experiência não-heteronormativa. Entretanto, apontamos ser uma limitação conceitual que deve ser superada, já que coloca a heterossexualidade enquanto modelo, e outras experiências afetivas como alteridade em relação à norma.
}

outra é dos estudos que tomam como foco as práticas sexuais das mulheres, utilizando os termos: mulheres que fazem sexo com mulheres (MSM), mulheres que fazem sexo com homens e mulheres (MSHM), mulheres de práticas homoeróticas e mulheres de práticas bissexuais.

A segunda categoria é utilizada para incluir na discussão mulheres que não se identificam como não-heterossexuais ${ }^{2}$ ou qualquer outra orientação sexual, mas que se relacionam sexualmente com outras mulheres. Além disso, também é utilizada para abranger mulheres das mais diversas orientações sexuais em uma mesma caracterização. Há discussões quanto ao uso desses termos comportamentais, pois ao não considerarem a orientação sexual, acabam generalizando a categoria e subalternizando uma série de aspectos relacionados a vivências não-heterossexuais específicas, que influenciam no contexto de prevenção às ISTs/HIV (como estigma por conta da orientação sexual).

Há uma discrepância com relação a orientação, práticas e desejos sexuais nesse grupo de mulheres, demonstrando não serem esferas fixas ou em consonância. No estudo de Mora e Monteiro (2013), poucas mulheres que se autodeclararam lésbicas possuíam trajetória sexual exclusiva com mulheres, semelhante ao que Herrick, Kuhns, Kinsky, Johnson e Garofalo (2013), Lindley, Walsemann e Carter Jr (2013) e Logie, Navia e Loutfy (2015) encontraram. Nesse contexto, "as experiências sexuais com homens adquirem diversos significados e nem sempre são associadas à identidade bissexual"' (Mora \& Monteiro, 2013, p. 907).

Além disso, constatamos uma fluidez na orientação, expressão e desejo sexual na trajetória de vida dessas mulheres (Mora \& Monteiro, 2013). É também por conta desse 
complexo cenário que os estudos utilizam diversos termos, e isso deve ser considerado nas discussões dessa temática, assim como na construção de espaços de acolhimento nos serviços de saúde.

Compreendendo esse cenário de fluidez e incongruência, optamos por utilizar o termo mulheres cisgêneras de vivências nãoheteronormativas, para se referir a mulheres de orientações, práticas e culturas sexuais nãoheteronormativas. $\mathrm{O}$ conceito vivências se refere a movimento, a uma não-fixidez, que apesar de se materializar em ato, ainda é dinâmica em sua essência. Cisgêneras é por conta desses estudos apenas considerarem mulheres que se identificam com o gênero que lhes foi atribuído no nascimento (Jesus, 2012), sendo mulheres que possuem vulva. $\mathrm{O}$ termo não-heteronormativas é para se referir às mulheres de diversas orientações sexuais, e também aquelas que não se identificam com nenhuma, mas que possuem práticas nãoheteronormativas que as vulnerabilizam no contexto preventivo entre mulheres. Apesar de utilizarmos esse termo, é necessário ressaltar a importância de considerar a vivência singular de cada orientação sexual, pois a partir delas e de outros marcadores sociais entrecruzados, cenários de vulnerabilidade são construídos de formas distintas.

Apontamos que a transição da utilização do termo "lésbicas", "bissexais", "queer" e outros para "mulheres cisgêneras de vivências não-heteronormativas" visa ressaltar a multiplicidade de expressões da sexualidade humana e suas formas de fluidez, sugerindo a necessidade de que o campo da prevenção não seja pensado apenas a partir de categorias fixas.

\section{Estigma}

Estigma é a demarcação de uma diferença, a partir da qual se desqualifica o sujeito ou grupo de sujeitos. A discriminação está vinculada ao estigma, e significa tratar de forma desigual indivíduos estigmatizados (como negando acesso a algo, por exemplo) (UNAIDS, 2017). Há ainda a violência, que pode ser uma ação desencadeada a partir da discriminação de determinado grupo, sendo a agressão sexual uma de suas formas.

Esses aspectos estão associados ao aumento da vulnerabilidade de determinados grupos populacionais às ISTs/HIV. Por conta da violência com base em gênero, mulheres são um grupo mais propenso a ser violentado (Daly, Spicer \& Willan, 2016; Logie, Navia, Rwigema, Tharao, Este \& Loutfy, 2014b) e constituírem vivências de vulnerabilidade, que estatisticamente são ainda potencializadas em mulheres não-heterossexuais por conta da discriminação por orientação sexual. Como exemplo, existe o denominado 'estupro corretivo', termo utilizado para caracterizar comumente de forma sensacionalista - os estupros de mulheres lésbicas com base na ideia de estar "corrigindo" a orientação sexual delas (Daly et al., 2016; Lindley, Walsemann \& Carter Jr., 2013; Logie, Alaggia, \& Rwigema, 2014a; Muranda et al., 2014; Palma \& Orcasita, 2017; Poteat et al., 2015).

Em estudo com mulheres de minoria sexual de Toronto, $41,7 \%$ delas relataram já terem tido alguma experiência de agressão sexual, sendo que $20,7 \%$ já tiveram alguma IST. Além disso, o histórico de sexo forçado foi associado com escasso apoio social e familiar e baixa autoestima (Logie et al., 2014a). Daly, Spicer e Willan (2016) apontam que, por mais urgente que seja esse contexto para a saúde, ainda há mais sensacionalismos sobre essa questão do que discussões públicas para seu enfrentamento, não sendo tratado como um problema de saúde pública.

As participantes do estudo de Logie, Alaggia e Rwigema (2014b), que se identificam como queer, relataram significativamente mais experiências de agressão sexual do que as que se identificam como lésbicas. As autoras pautam-se em Austin, Roberts e Corliss (2008) para discutir esse contexto, apontando que os motivos que tangem esse dado podem estar relacionados com: formação da orientação sexual - com a violência podendo mudar a forma como a pessoa se identifica -, congruência de relato e apoio social de grupo identitário, sendo que este pode diminuir a 
vulnerabilidade. Esse dado demonstra a importância de considerar diversas orientações sexuais.

Logie et al., (2015) constataram que experiências de estigma sexual ${ }^{3}$ foram associadas com 6.5 vezes mais relatos de histórico de ISTs em amostra com MSM. No estudo de Poteat et al. (2015) com MSM de Lesoto, $74 \%$ relataram pelo menos uma experiência de estigma sexual ${ }^{4}$. Nesse mesmo estudo, o estigma no trabalho teve a maior associação com a soropositividade, uma vez que aquelas que perderam o emprego obtiveram 15 vezes mais chances de reportar diagnóstico de HIV. Além disso, houve diversos relatos de estigma no setor de saúde, judiciário e no ciclo familiar e comunitário.

\section{Outra violência mencionada nos} estudos e que foi relacionada com vulnerabilidade às ISTs/HIV, é a violência por parceira/o íntimo/a (VPI). Quase metade (40\%) da amostra de MSM e MSHM afro-americanas do estudo conduzido por Muzny, Austin, Harbison e Hook (2014a) relataram ter vivenciado tal violência. Segundo Palma e Orcasita (2017), há diversos fatores que contribuem para a escassa atenção e número de denúncias oficiais dessa questão, tais como "a má aplicação da lei em violência por parceira/o íntima/o em relações de mesmo sexo, escasso apoio social, estigma dentro do sistema jurídico e centros de cuidado à saúde; e baixa percepção social e individual sobre o tema" (p. 1033). Destacamos a necessidade de mais investigações da relação dessa violência com a vulnerabilidade às IST-HIV, além da variância no número de casos a partir de diferentes marcadores sociais.

Esses dados apontam para a necessidade das ações em saúde também sensibilizarem o setor judiciário e da polícia com relação à violência praticada contra

\footnotetext{
${ }^{3}$ A palavra estigma nesse estudo e no estudo de Poeat et al. (2015) é utilizada para se referir a violências geradas a partir do estigma.
}

mulheres cisgêneras de vivências nãoheteronormativas, pois a discriminação dentro desses setores limita o acesso a denúncias, apoio social e, consequentemente, acesso a saúde e seus direitos humanos fundamentais (Daly et al., 2016; Poteat et al., 2015). A proteção legal pode ser vista como um elemento importante na prevenção de ISTs/HIV nesse grupo (Poteat et al., 2015). Ademais, programas que visam promover saúde sexual para essas mulheres devem incluir estratégias que desenvolvam redes de apoio social (Palma \& Orcasita, 2017), além de considerarem cuidados específicos para mulheres que sofreram agressão sexual (Logie et al., 2014a).

\section{Despreparo das/os profissionais de saúde}

Diversos estudos apontam para um despreparo das/os profissionais de saúde no atendimento de mulheres lésbicas, bissexuais e outras mulheres cisgêneras de vivências nãoheteronormativas. As experiências no setor de saúde relatadas pelas mulheres eram de caráter heteronormativo e prescritivo, com orientações descontextualizadas (Batista \& Zambenedetti, 2017; Carvalho et al., 2013; Muranda et al., 2014; Muzny et al., 2013; Poteat et al., 2015). Além disso, elas apontaram para uma percepção de descaso por parte das/os profissionais quando relatado sexo com outras mulheres, seja por não mudarem a abordagem heterocêntrica do atendimento ou por deixarem de solicitar exames importantes, como de ISTs/HIV e o Papanicolau (Agénor, Muzny, Schick, Austin \& Potter, 2017; Batista \& Zambenedetti, 2017; Carvalho et al., 2013; Muranda et al., 2014; Silberman et al., 2016).

Esse cenário é atravessado pela ideia de imunidade lésbica, presente no imaginário social, que banaliza o sexo lésbico. Ao considerarem o sexo entre mulheres como uma 'brincadeira', um não-sexo ou um sexo não tão

\footnotetext{
${ }^{4}$ Nesse estudo, as experiências de estigma sexual mais relatadas foram: assédio verbal ou psíquico (52\%), chantagem (29\%), apanhar por conta da orientação sexual (14\%) e estupro (9\%). Entretanto, 23\% não responderam à questão do estupro. $\mathrm{N}=250 \mathrm{MSM}$. (Poteat et al., 2015)
} 
autêntico quanto o heterossexual, cria-se o imaginário de que não é possível transmitir ISTs/HIV por essas práticas (Mora \& Monteiro, 2013; Muranda et al., 2014; Muzny et al., 2013). Essa questão também é um dos resultados da invisibilidade lésbica e de outras mulheres cisgêneras de vivências nãoheteronormativas, sendo necessário, como um primeiro passo, fazer visível essas vivências e demandas (Carvalho et al., 2013).

Por conta de experiências de discriminação dentro e fora do setor de saúde, essas mulheres relataram medo e desconforto ao procurar serviços de saúde (Carvalho et al., 2013, Muranda et al., 2014; Muzny et al., 2013; Poteat et al., 2015). No estudo de Poteat et al (2015), além de $22.3 \%$ das mulheres relatarem esse medo, $23 \%$ apontaram ouvir trabalharoras/es fofocando sobre elas. Isso implica: no afastamento das mulheres em relação a estes; na criação de um imaginário no qual não há necessidade de um acompanhamento de saúde; no desconhecimento do próprio corpo; e poucas práticas de autocuidado (Batista \& Zambenedetti, 2017; Carvalho et al., 2013; Lindley et al., 2013; Muranda et al., 2014). Além disso, diagnósticos de ISTs foram associados com atendimento de saúde negado no estudo de Poteat et al. (2015), e crença de que a/o profissional de saúde se sentiu desconfortável com a orientação sexual no estudo de Logie et al. (2015). O único estudo que diferiu nessa questão foi o de Silberman, Buedo e Burgos (2016), no qual $81,1 \%$ das mulheres não perceberam preconceito no atendimento médico. Entretanto, a maioria das participantes eram universitárias, podendo ser um aspecto que interfere no resultado.

Alguns artigos mencionam alternativas para profissionais de saúde reduzirem a lacuna no atendimento de mulheres cisgêneras com vivências não-heteronormativas, prestando serviços qualificados e inclusivos. Entre essas alternativas estão: conhecer a trajetória sexual das mulheres no atendimento - considerando a diversidade de suas práticas sexuais e a fluidez e incongruência entre práticas e orientações sexuais (Herrick, Kuhns, Kinsky, Johnson \& Garofalo, 2013; Logie et al., 2014a; Mora \& Monteiro, 2013; Muzny et al., 2013); informarse sobre comportamentos que podem transmitir ISTs nesse grupo; recomendar o uso de camisinha em brinquedos sexuais e no sexo com parcerias masculinas; encorajar o uso e adaptações de métodos preventivos com os quais elas se sintam contempladas no sexo com mulheres (como a realização de testes de IST); e fornecer mensagens que ligam barreiras ao prazer (Muzny et al., 2013; Doull et al., 2017), como:

Por exemplo, a borda externa do preservativo feminino pode estimular o clitóris durante o sexo, ou a lubrificação usada na parte inferior de um dental dam $^{5}$ pode aumentar o prazer para a mulher que recebe sexo oral. (Doull et al., 2017, p.415).

Essas/esses profissionais também devem considerar outros contextos específicos para um atendimento qualificado, como histórico de agressão sexual (Logie et al., 2014a). Entre outras questões que os estudos apontam estão: a importância de considerar que algumas mulheres não tiveram experiência de penetração na vida sexual, ou não se sentem confortáveis sendo penetradas; não presumir a sexualidade a partir de características pessoais - normalmente ligadas a estereóripos de gênero (Carvalho et al., 2013); não suporem que o sexo entre mulheres se resume ao sexo oral, direcionando o atendimento para essa prática (Muzny et al., 2013); e considerar o contexto diferenciado de mulheres bissexuais (Logie, Navia \& Loutfy, 2015), que será abordado adiante nesse trabalho.

\footnotetext{
5 Dental dam é um material de látex em forma de quadrado usado por dentistas, mas que pode funcionar como um método de barreira entre a língua e a pele no sexo oral.
} 
Apesar de grande parte dos estudos apontarem para o despreparo profissional no atendimento dessas mulheres, nenhum deles foi realizado com as/os próprias/os profissionais.

\section{(Des)conhecimento x Conhecimento impreciso x Práticas de prevenção}

Além de refletir no despreparo das/os profissionais de saúde, a falta de discussão sobre o tema também afeta no conhecimento das próprias mulheres sobre sua saúde sexual. Amostras de mulheres cisgêneras com vivências não-heteronormativas de diversos artigos analisados relataram um descaso com relação a prevenção, seja por considerarem o risco de transmissão às ISTs/HIV no sexo entre mulheres como baixo ou inexistente; por não saberem ao certo quais ISTs estão relacionadas com essas práticas; ou pelo desconhecimento de possíveis métodos preventivos (Carvalho et al., 2013; Doull et al., 2017; Muranda et al., , 2014; Muzny et al., 2013; Palma \& Orcasita, 2017; Silberman et al., 2016). Como exemplo, no estudo de Muranda, Mugo e Antonites (2014), 46\% das mulheres demonstraram não saber onde procurar informações sobre a utilização de métodos de barreira no sexo entre mulheres, sendo que $61 \%$ acessaram essas informações através da internet.

Há ainda uma lacuna com relação a aplicabilidade desses métodos. Mulheres que relataram estarem cientes do risco em relação às ISTs/HIV em suas relações sexuais com outras mulheres e também conhecerem alguns métodos preventivos, demonstraram desamparo quanto a adesão destes em suas práticas sexuais, seja por incômodo, por considerarem que há poucas opções disponíveis ou por não saberem ao certo como usá-los ou onde encontrá-los (Batista \& Zambenedetti, 2017; Muzny et al., 2013). Destacamos a importância dos serviços de saúde informarem onde é possível encontrar métodos de barreira, além da disponibilização dos mesmos através de políticas públicas. A falta de desenvolvimento de métodos adequados e específicos para o sexo entre mulheres também é um aspecto importante nesse contexto, pois as alternativas conhecidas e aplicadas por elas são considerados improvisos (Carvalho et al., 2013). Dessa forma, além de políticas e intervenções, devem ser desenvolvidos materiais que atendam as demandas específicas desse público, e que sejam disseminadas para o acesso a informações e métodos de prevenção pelo próprio grupo (Batista \& Zambenedetti, 2017; Carvalho et al., 2013).

Para além do desamparo, as barreiras são ainda consideradas componentes que afetam na obtenção de prazer (Batista \& Zambenedetti, 2017; Doull et al., 2017; Silberman et al., 2016). A necessidade do toque e do sabor das secreções vaginais foi colocada pelas participantes dos estudos como importante componente do interesse sexual, além de mencionarem que a utilização dos métodos afetaria na dinâmica da relação sexual (Batista \& Zambenedetti, 2017; Doull et al., 2017). O estudo de Doull et al. (2017) incluiu mulheres experientes e não-experientes sexualmente em sua amostra, e o segundo grupo visualizou algumas vantagens do uso de barreiras que o outro não mencionou - como o desing de camisinhas para aumentar o prazer e ainda demonstraram maior abertura para considerarem a utilização de barreiras. Dessa forma, fornecer informações antes da iniciação sexual pode aumentar o uso de barreiras entre essas mulheres.

Alguns comportamentos e métodos preventivos citados por esses estudos foram: cuidados básicos de higiene, como lavar as mãos e manter as unhas cortadas; adaptação da camisinha masculina e uso da camisinha de língua; uso de luvas descartáveis/luvas látex e soluções microbianas tópicas em gel - que possibilitam diminuir a transmissão por secreção vaginal; uso de preservativos masculinos em brinquedos sexuais e sua posterior higienização; uso de dental dams e plástico filme; camisinha feminina; realização de testes de ISTs e exames de rotina; e evitar sexo oral durante a menstruação (Batista \& Zambenedetti, 2017; Carvalho et al., 2013; Muranda et al., , 2014; Muzny et al., 2013).

Os métodos mais bem aceitos pelas mulheres foram a realização de testes e 
cuidados básicos de higiene (Muzny et al., 2013), sendo que o primeiro foi colocado por Doull et al. (2017) como um dos fatores que fazem com que as mulheres não utilizem métodos de barreira. Entretanto, no estudo de Muranda et al. (2014), participantes lésbicas relataram se sentirem desconfortáveis em realizar testes para ISTs por conta do imaginário que mulheres lésbicas reais são imunes, sendo um aspecto que influencia na vulnerabilidade desse grupo. $\mathrm{O}$ uso de dental dams e luvas látex foi considerado por algumas participantes do estudo de Muzny, Harbison, Pembleton e Austin (2013) como útil, porém não viável para uso. Participantes do estudo de Batista e Zambenedetti (2017) questionaram se o desconforto em utilizar métodos preventivos pode ser resultado do apagamento da mulher lésbica e bissexual nas ações de prevenção, sendo necessária a criação de uma cultura preventiva, na qual haja possibilidades de práticas que relacionam prazer com prevenção. Afinal, como será possível criar uma relação de confiança e prazer com métodos de barreira se não há informações sobre a existência de mulheres que fazem uso desses materiais?

Ademais, muitas mulheres relataram que acessaram informações de prevenção em espaços online da comunidade LGBT, ou em suas relações pessoais (Batista \& Zambenedetti, 2017; Muranda et al., 2014; Silberman et al., 2016). Por se constituir como um espaço de criação de estratégias e discussões, a disseminação de informações pela internet pode ser uma alternativa para mais mulheres aderirem a métodos preventivos (Muranda et al., 2014). Entretanto, é importante ressaltar que isso abrange apenas um grupo de mulheres: as que possuem acesso à internet. Insere ainda outro desafio, que é o da constituição de referências seguras em meio a diversidade de informações disponíveis online.

Outra questão que demonstrou permear o não-uso de barreiras por essas mulheres nos estudos é a confiança na parceria sexual ou possuir parceria fixa (Carvalho et al., 2013; Doull et al., 2017; Mora \& Monteiro, 2013; Silberman et al., 2016). Como exemplo, no estudo de Silberman et al. (2016), no qual
$77,5 \%$ das mulheres que fazem sexo com mulheres que conhecem métodos preventivos dizem não os usar por: parceria fixa $(39,7 \%)$, incômodo $(23,3 \%)$ ou porque não conseguem $(17,8 \%)$. Além disso, muitas mulheres demonstraram se considerarem mais vulneráveis no sexo com homens, principalmente por ligarem transmissão a prática penetrativa (Carvalho et al., 2013; Doull et al., 2017; Muzny et al., 2013).

\section{Percepções acerca de mulheres bissexuais e outras MSHM}

Há uma série de percepções construídas acerca de mulheres bissexuais que impactam no contexto preventivo deste grupo e de mulheres lésbicas. No imaginário destas, segundo estudo realizado em um bairro do Rio de Janeiro, se relacionar com mulheres de identidade bissexual implica em insegurança, relacionada também à transmissão de HIV (Mora \& Monteiro, 2013). Alguns estudos apontam que isso está relacionado com a ideia de que os homens e a prática penetrativa estão ligadas à transmissão, e as ISTs são classificadas como agentes que 'infiltram' na comunidade lésbica (Muranda et al., 2014; Muzny et al., 2013). Isso também está relacionado com a ideia de imunidade lésbica e banalização do sexo lésbico abordada anteriormente, além do imaginário que orientação sexual e práticas sexuais são esferas fixas e congruentes.

Essa percepção de mulheres lésbicas faz parte do que Leite e Luna (2002) chamam de proteção imaginária. Esta é resultado de justificativas (nem sempre conscientes) construídas para a não adesão de métodos preventivos. Mesmo com o estudo não citando mulheres lésbicas, esse conceito se atualiza nas percepções construídas por essas mulheres. Em parte, a proteção imaginária nesse contexto é amparada pela ciência, pois esta afirma que existe "menor" probabilidade de infecção nas relações entre mulheres.

Relacionar ISTs e HIV com mulheres bissexuais, reflete em ações e pensamentos preconceituosos com relação à estas (Muzny et al., 2013), e também acaba influenciando na 
vulnerabilidade de mulheres lésbicas no contexto de prevenção às ISTs/HIV. Ademais, isso dificulta a discussão de experiências de violência sexual e estupro, já que lésbicas podem acabar contraindo ISTs por conta dessa violência (Muranda et al., 2014).

Estudos também apontam que mulheres bissexuais ou MSHM estão mais propensas a vivenciarem contextos que potencializam a vulnerabilidade às ISTs/HIV, relacionando alguns comportamentos com mais diagnósticos de ISTs nesse grupo (Estrich, Gratzer \& Hotton, 2014; Herrick et al., 2013; Lindley et al., 2013; Muzny et al., 2013; Muzny, Austin, Harbison \& Hook, 2014a; Muzny, et al. 2014b). Entre eles estão: maior número de parcerias sexuais, de parcerias casuais/novas e parcerias masculinas; sexo em grupo; sexo com pessoas que possuem alguma IST; e prostituição (Lindley et al., 2013; Muzny et al., 2013; Muzny et al., 2014a; Muzny et al., 2014b).

No estudo de Bostwick, Hughes e Everett (2015), comparado com mulheres lésbicas, mulheres bissexuais relataram mais uso de cocaína e experiências de ansiedade, além de marcarem mais pontos em escala de depressão. No estudo citado, elas não diferiram em renda e idade, diferente do que Muzny et al. (2014a) e Agénor, Muzny, Schick, Austin e Potter (2017) encontraram, sendo necessário mais investigações quanto a esse aspecto. Mulheres bissexuais também foram mais propensas a terem sido violentadas com sexo vaginal forçado por um homem no estudo de Agénor et al. (2017).

Schick, Pol, Dodge, Baldwin \& Fortenberry (2015) apontaram a realização de autoamostragem como alternativa para aumentar a adesão de mulheres de práticas bissexuais a testes de ISTs. Nesse estudo, o método foi bem aceito pelas mulheres, podendo contribuir para a expansão das possibilidades de testagem e redução das chances de vivenciar discriminação no setor de saúde.

\footnotetext{
${ }^{6}$ Nation Survey on Drug Use and Health.
}

\section{Raça/cor}

Outros dois grupos que foram significantemente mencionados nos estudos da amostra deste trabalho foram mulheres da África do Sul e mulheres afro-americanas. Agrupamos em uma mesma linha de análise por despertarem discussões similares, colocando em evidência o marcador raça/cor. A África do Sul possui uma das maiores incidências de HIV do mundo, além de apresentar elevados índices de discriminação por orientação sexual. Isso advém das normas sociais conservadoras do país, agravando a vulnerabilidade às ISTs/HIV em grupos considerados minoritários (Daly et al., 2016; Muzny et al., 2014a). Nesse mesmo contexto, há diferenças no número de MSM que vivem com HIV a partir de raça, com índices mais elevados em mulheres negras do que brancas (Daly et al., 2016). Além disso, Muranda et al. (2014) apontam para um estudo que menciona que $6,6-9,6 \%$ de mulheres que se identificam como lésbicas ou bissexuais na África do Sul são soropositivas, ressaltando ser um número significante para um grupo visto como não-vulnerável.

No estudo de Herrick et al. (2013), ser mulher afro-americana foi associado com mais diagnósticos de ISTs. Muzny et al. (2014a) apontam ainda que estas que fazem sexo com homens e mulheres se inserem em maior proporção em contextos ditos de comportamento sexual de risco do que MSM do mesmo grupo - como maior número de parcerias sexuais e parcerias masculinas, sexo em grupo e parcerias que possuem alguma IST. Também, o número de ISTs curáveis e nãocuráveis entre MSM e MSHM brancas é menor do que em afro-americanas, especialmente em MSHM.

Além disso, Muzny et al. (2014a) constataram que o uso de drogas ilícitas entre mulheres afro-americanas de minorias sexuais foi significantemente maior (32\% MSM e $41 \%$ MSHM) do que em mulheres africanas em um geral $(6,7 \%)^{6}$. Essas constatações demonstram como não é possível analisar orientação sexual, 
raça, classe e fatores sociodemográficos de forma indissociada em uma análise complexa de fenômenos que agravam a vulnerabilidade às ISTs/HIV. Muzny et al. (2014a), mesmo não mencionando o conceito de interseccionalidade, apontam para o que chamam de "risco triplo" (p.611) na experiência de mulheres afro-americanas, já que o sexo, a raça e a orientação sexual dessas mulheres são marcadores que interferem no exercício a saúde.

Os estudos não possibilitaram compreender se há diferença na percepção de risco de transmissão às ISTs e na vivência de atendimentos desqualificados no setor de saúde entre mulheres negras, afro-americanas e da África do Sul e mulheres brancas ou de outros países. A vulnerabilidade é potencializada no sentido de desencadear contextos mais propensos a experiências de discriminação, além de condições socioeconômicas mais precárias. Apontamos para a necessidade de mais investigações quanto ao contexto específico dessas mulheres.

\section{Conscientização política}

A partir dos estudos é possível compreender que um elemento chave para que a saúde sexual de mulheres cisgêneras com vivências não-heteronormativas se torne uma pauta visível no social e no campo da saúde - e que suas sexualidades possam ser exercidas de forma digna - é a conscientização política em relação ao tema e a identidade lésbica, bissexual ou outra. Criar estratégias de empoderamento $^{7}$ e reivindicação de direitos junto à essas mulheres pode contribuir para que estas se sintam encorajadas a buscar serviços de saúde e exigir que suas demandas sejam atendidas, além de fornecer meios para a conscientização de seus direitos humanos

\footnotetext{
${ }^{7}$ Segundo Baquero (2012), o empoderamento “[...] pode ser concebido como emergindo de um processo de ação social no qual os indivíduos tomam posse de suas próprias vidas pela interação com outros indivíduos, gerando pensamento crítico em relação à realidade, favorecendo a construção da capacidade pessoal e social e possibilitando a transformação de relações sociais de poder" (p.181).
}

fundamentais. Ademais, construir redes coletivas de enfrentamento à discriminação e violência, no qual possa ser fornecido um espaço de compartilhamento de experiências relacionadas a orientação e práticas sexuais, pode, também, diminuir a vulnerabilidade às ISTs/HIV (Coulter, Kinsky, Herrick, Stall \& Bauermeister, 2015; Muranda et al., 2014; Palma \& Orcasita, 2017; Poteat et al., 2015). Para Coulter, Kinsky, Herrick, Stall \& Bauermeister (2015), as intervenções no campo da saúde devem focar em diminuir a discriminação e aumentar o enfrentamento saudável, já que isto está relacionado com a diminuição dos ditos comportamentos sexuais de risco, sintomas de depressão e uso de substâncias nesse grupo.

Ao discutir direito à saúde, Muranda et al. (2014) apontam para a comunicação Purohit and Moore v. The Gambia da Comissão Africana dos Direitos Humanos e dos Povos. Esta afirma que o direito à saúde não está somente ligado ao direito de acessar os serviços de saúde, e sim de criar ferramentas que promovam esse acesso. Dessa forma, exercer direito à saúde é poder ter acesso a informações qualificadas sobre o que se demanda e necessita.

Assim, é necessário que sejam desenvolvidas intervenções, programas e políticas que visem promover saúde sexual para essas mulheres, levando em conta a implicação dos contextos específicos abordados neste trabalho. Alguns estudos apontam ações específicas necessárias, como: o desenvolvimento de acesso à educação sexual (Herrick et al., 2013); criação de estratégias diante de violência sexual e problemas familiares, junto com a construção de redes de apoio e desenvolvimento de competência de profissionais para apoiarem e auxiliar essas 
mulheres (Logie et al., 2014); explorar estratégias de estilos de enfrentamento considerando as diversas orientações sexuais (Logie et al., 2014a); criação de programas voltados para a conscientização de transmissão de ISTs e índices de câncer cervical entre mulheres que se relacionam com mulheres (Agénor et al., 2017); e integração do tema nos currículos de medicina e outros campos da saúde (Silberman et al., 2016).

\section{Outros aspectos relacionados a vulnerabilidade}

Entre outros aspectos relacionados à vulnerabilidade às ISTs/HIV estão: ter várias parcerias sexuais; sexo sob efeito de álcool e drogas; sexo vaginal sem proteção; sexo anal; possuir relações sexuais com homens e mulheres; status de relacionamento; alto risco de pensamentos e tentativas suicidas; e sintomas de depressão (Coulter et al., 2015; Herrick et al., 2013; Lindley et al., 2013; Logie et al., 2015; Palma \& Orcasita, 2017). Além disso, é necessário considerar o número elevado de prisão, encarceramento e instabilidade social nesse grupo (Palma \& Orcasita, 2017).

O uso de drogas injetáveis e a condição social-econômica também apareceram associadas à vulnerabilidade ${ }^{8}$. German e Latkin (2015) investigaram se há disparidades entre instabilidade social e vulnerabilidade ao HIV relacionado com orientação sexual. Nesse estudo, as mulheres de minorias sexuais foram três vezes mais propensas a relatarem sexo por troca de dinheiro ou drogas (prostituição), e quatro vezes mais propensas a relatarem uma IST recente do que mulheres heterossexuais. Além disso, reportaram mais encarceramento estendido nos últimos 10 anos; e 60\% delas passou pelo menos 6 meses encarcerada, comparado com $24 \%$ das heterossexuais. A

\footnotetext{
${ }^{8} \mathrm{O}$ termo utilizado pelo artigo era mulheres usuárias de drogas injetáveis (UDI). Entretanto, por conta do entendimento que esse termo desumaniza o grupo, optamos por utilizar a nomenclatura recomendada pelo Guia de Terminologia da UNAIDS (2017).
}

amostra do artigo é predominantemente afroamericana $(83 \%)$.

\section{Considerações Finais}

O conceito de vulnerabilidade apresentou-se como uma importante ferramenta de análise do contexto de prevenção às ISTs/HIV entre mulheres lésbicas, bissexuais e outras mulheres cisgêneras de vivências não-heteronormativas. Além disso, concluímos que a vulnerabilidade deve ser analisada a partir de diferentes orientações sexuais - como lésbicas, bissexuais ou queer -, além de outros marcadores sociais, como a raça e a classe. Indicamos ainda que novos estudos possam avaliar a relação de outros marcadores - como o geracional - com a vulnerabilidade, já que nenhuma amostra dos artigos analisados incluiu mulheres idosas. Indicamos, como limitação deste estudo, a restrição da pesquisa a duas bases de dados. Também, faz-se necessário o desenvolvimento de mais estudos na América Latina, já que apenas quatro artigos foram feitos e publicados nesse território. Ademais, apesar dos estudos destacarem marcadores que interagem no processo de vulnerabilidade, o conceito de interseccionalidade ainda está pouco presente, podendo ser mais explorado em novos trabalhos.

Mesmo com diversos artigos apontando para a necessidade da sensibilização de equipes de saúde, nenhuma das pesquisas foi realizada com estas. Nesse sentido, indicamos a necessidade da realização de pesquisas com profissionais de saúde com o intuito de ampliar a compreensão acerca de suas percepções sobre a abordagem preventiva com o público lésbico e bissexual. Além de pesquisas, apontamos para a necessidade de políticas e programas de formação profissional que abordem o tema da prevenção no sexo entre mulheres. Ademais, apesar de haver diversos estudos que apontam 
para a consideração do contexto específico de mulheres bissexuais, somente uma pesquisa possuía como amostra exclusivamente mulheres de práticas bissexuais. Destacamos também a importância de estudos que considerem a existência de mulheres lésbicas e bissexuais transexuais, tirando do foco da prevenção apenas o caráter genitário.

Indicamos ainda a realização de estudos com mulheres que se relacionam sexualmente com mulheres e são soropositivas. Compreender a especificidade desse contexto pode, além de promover apoio e visibilidade para esse grupo, investigar possíveis métodos de barreira. Pesquisas com amostras de mulheres lésbicas e bissexuais que se previnem também pode contribuir com esse objetivo e auxiliar no desenvolvimento de abordagens e métodos que relacionam sexo, cuidado, prazer e prevenção.

A construção de uma consciência política sobre direitos sexuais por essas mulheres e junto a elas também demonstrou ser um elemento chave na diminuição da vulnerabilidade às ISTs/HIV. Apontamos, por fim, a necessidade de romper com o silenciamento em torno da prevenção entre mulheres lésbicas e bissexuais, através do desenvolvimento de políticas públicas, programas e intervenções que abordem saúde sexual e prevenção de ISTs/HIV entre esse grupo, considerando a complexidade de seus contextos e a diversidade de vivências a partir do atravessamento de diferentes marcadores sociais.

\section{Referências}

Agénor, M., Muzny, C. A., Schick, V., Austin, E. L., \& Potter, J. (2017). Sexual orientation and sexual health services utilization among women in the United States. Preventive Medicine, 95, 74-81. doi: 10.1016/j.ypmed.2016.11.023

Ayres, J. R. C. M., Junior, I. F., Calazans, G. J., \& Saletti, H. C. F. (2009). O conceito de vulnerabilidade e as práticas de saúde: novas perspectivas e desafios. In: D. Czeresnia, C. M. Freitas, Promoção da saúde: conceitos, reflexões, tendências (pp. 121-144). Rio de Janeiro, FIOCRUZ.

Baquero, R. V. A. (2012). Empoderamento: instrumento de emancipação social? - Uma discussão conceitual. Revista Debates, 6(1), 173-187. doi: $10.22456 / 1982-5269.26722$

Barbosa, B. R. S. N., Nascimento, E. T., Carvalho, I. A. F., \& Cavalcante, J. C. D. (2014). Invisibilidade lésbica e a interseccionalidade de opressões. In Anais do 18 Redor, (pp. 3008-3024). Recife, PE: Universidade Federal Rural de

Pernambuco. Recuperado de: http://www.ufpb.br/evento/index.php/18red or/18redor/paper/viewFile/2269/821

Batista, M. C. H., \& Zambenedetti, G. (2017). Uma pesquisa-intervenção sobre prevenção às IST/HIV com mulheres lésbicas e bissexuais. Psicol. Pesq, 11(2), 42-60. doi: 10.24879/2017001100200180

Bostwick, W. B., Hughes, T. L., \& Everett, B. (2015). Health Behavior, Status, and Outcomes Among a Community-Based Sample of Lesbian and Bisexual Women. LGBT Health, 2(2), 121-126. doi: 10.1089\%2Flgbt.2014.0074

Carrara, S. (2010). Políticas e direitos sexuais no Brasil contemporâneo. Bagoas, 4(5), 131-147. Recuperado de https://periodicos.ufrn.br/bagoas/article/vie w/2316

Carvalho, P. M. G., Nóbrega, B. S. M., Rodrigues, J. L., Almeida, R. O., Abdalla, F. T. M. \& Nichiata, L. Y. I. (2013). Prevención de enfermidades de transmisión sexual por mujeres homosexuales y bisexuales: estudio descriptivo. Online Brazilian Journal of Nursing, 12(4). Recuperado de: http://www.objnursing.uff.br/index.php/nur sing/article/view/4177/html_37

Coulter, R. W. S., Kinsky, S. M., Herrick, A. L., Stall, R. D., \& Bauermeister, J. A. (2015). Evidence of Syndemics and Sexuality-Related Discrimination Among 
Young Sexual-Minority Women. LGBT Health, 2(3), 250-257. doi: 10.1089/lgbt.2014.0063

Daly, F., Spicer, N., \& Willan, S. (2016). Sexual rights but not the right to health? Lesbian and bisexual women in South Africa's National Strategic Plans on HIV and STIs. Reproductive Health Matters, 24(47), 185-194. doi:

10.1016/j.rhm.2016.04.005

Doull, M., Wolowic, J., Saewyc, E., Rosario, M., Prescott, T., \& Ybarra, M. L. (2017). Why Girls Choose Not to Use Barriers to Prevent Sexually Transmitted Infection During Female-to-Female Sex. Journal of Adolescent Health, 62(4), 411-416. doi: 10.1016/j.jadohealth.2017.10.005

Estrich, C. G., Gratzer, B., \& Hotton, A. L. (2014). Differences in sexual health, risk behaviors, and substance use among women by sexual identity: Chicago, 20092011. American Sexually Transmitted Diseases Association, 41(3), 194-199. doi: 10.1097/olq.0000000000000091

Facchini, R., \& Barbosa, R. M. (2006). Dossiê: Saúde das Mulheres Lésbicas Promoção da equidade e da integralidade. Belo Horizonte: Rede Feminista de Saúde.

German, D., \& Latkin, C. A. (2015). HIV Risk, Health, and Social Characteristics of Sexual Minority Female Injection Drug Users in Baltimore. AIDS Behav, 19(7), 1361-1365. doi: 10.1007/s10461-014$\underline{0972-\mathrm{Z}}$

Gil, A. C. (2002). Como elaborar projetos de pesquisa (4a ed). São Paulo: Atlas.

Herrick, A., Kuhns, L., Kinsky, S., Johnson, A., \& Garofalo, R. (2013). Demographic, Psychosocial, and Contextual Factors Associated With Sexual Risk Behaviors Among Young Sexual Minority Women. Journal of the American Psychiatric Nurses Association, 19(6), 345-355. doi: $\underline{10.1177 / 1078390313511328}$

Hirata, H. (2014). Gênero, classe e raça: Interseccionalidade e consubstancialidade das relações sociais. Tempo Social, 26(1), 61-73. doi: $10.1590 /$ S0103$\underline{20702014000100005}$
Jesus, J. G. (2012). Orientações sobre a população transgênero: conceitos e termos. Brasília. Recuperado de:

https://www.scribd.com/document/8784652 6/Orientacoes-sobre-Identidadede-GeneroConceitos-e-Termos

Leite, R. M., \& Luna, E. (2002). O

Desenvolvimento do campo do conhecimento e da pesquisa em relação à infecção pelo HIV junto aos homossexuais e bissexuais. In Brasil, Novos Desafios da Prevenção da Epidemia pelo HIV/Aids junto aos homens que fazem sexo com homens (pp. 67-68). Brasília, DF: Ministério da Saúde.

Lindley, L. L., Walsemann, K. M., \& Carter Jr, J. W. (2013). Invisible and at Risk: STDs Among Young Adult Sexual Minority Women in the United States. Perspectives on Sexual and Reproductive Health, 45(2), 66-73. doi: 10.1363/4506613

Logie, C. H, Alaggia, R., \& Rwigema, M. J. (2014a). A social ecological approach to understanding correlates of lifetime sexual assault among sexual minority women in Toronto, Canada: results from a crosssectional internet-based survey. Health Education Research, 29(4), 671-682. doi: 10.1093/her/cyt119

Logie, C. H., Navia, D., Rwigema, M.-J., Tharao, W., Este, D., \& Loutfy, M. R. (2014b). A group-based HIV and sexually transmitted infections prevention intervention for lesbian, bisexual, queer and other women who have sex with women in Calgary and Toronto, Canada: study protocol for a non-randomised cohort pilot study. BMJ Open, 4(4), 1-7. doi: 10.1136/bmjopen-2014-005190

Logie, C. H., Navia, D., \& Loutfy, M. R. (2015). Correlates of a lifetime history of sexually transmitted infections among women who have sex with women in Toronto, Canada: results from a crosssectional internet-based survey. Sex Transm Infect, 91(4), 278-83. doi: 10.1136/sextrans-2014-051745

López, L. C. (2011). Uma Análise das Políticas de Enfrentamento ao HIV/Aids na Perspectiva da Interseccionalidade de Raça 
e Gênero. Saúde Soc, 20(3), 590-603. doi: 10.1590/S0104-12902011000300006

Ministério da Saúde. Brasil. (2013). Política Nacional de Saúde Integral de Lésbicas, Gays, Bissexuais, Travestis e Transexuais. Brasília - DF: Ministério da Saúde.

Mora, C. M., \& Monteiro, S. (2013).

Homoerotismo feminino, juventude e vulnerabilidade às DSTs/Aids. Estudos Feministas, 21(3), 905-926. doi: 10.1590/S0104-026X2013000300008

Muranda, T., Mugo, K., \& Antonites, C. (2014). HIV is not for me: A study of African women who have sex with women's perceptions of HIV/AIDS and sexual health. African Human Rights Law Journal, 14(2), 757-786. Recuperado de http://www.scielo.org.za/scielo.php?script= sci_arttext\&pid=S199620962014000200021\&lng=en\&tlng=en

Muzny, C. A., Austin, E. L., Harbison, H. S., \& Hook, E. W. (2014a). Sexual Partnership Characteristics of African American Women Who Have Sex With Women; Impact on Sexually Transmitted Infection Risk. Sexually Transmitted Diseases, 41(10), 611-617. doi: 10.1097/olq.0000000000000194

Muzny, C. A., Harbison, H. S., Pembleton, E. S., \& Austin, E. L. (2013). Sexual Behaviors, Perception of Sexually Transmitted Infection Risk, and Practice of Safe Sex Among Southern African American Women Who Have Sex With Women. Sexually Transmitted Diseases, 40(5), 395-400. doi: 10.1097/olq.0b013e31828caf34

Muzny, C. A., Rivers, C. A., Parker, C. J., Mena, L. A. Austin, E. L., \& Schwebke, J. R. (2014b). Lack of evidence for sexual transmission of genital Candida species among women who have sex with women: a mixed methods study. Sex Transm Infect, 90, 165-170. doi: 10.1136/sextrans-2013$\underline{051361}$

Palma, D. M., \& Orcasita, L. T. (2017). Considerations for the design of Human
Immunodeficiency Virus (HIV) prevention programs for lesbian and bisexual women. Interface (Botucatu), 21(63), 1031-1038. doi: $10.1590 / 1807-57622016.0790$

Peres, M. C. C., Soares, S. F., \& Dias, M. C. (2017). Dossiê sobre Lesbocídio no Brasil de 2014 até 2017. Rio de Janeiro: Livros Ilimitados.

Piscatelli, A. (2009). Gênero: A história de um conceito. In: H. B. de Almeida, \& J. Szwako (Orgs.), Diferenças, igualdade (pp. 116-146). São Paulo: Berlendis.

Poteat, T. C., Logie, C. H., Adams, D., Mothopeng, T., Lebona, J., Letsie, P., \& Baral, S. (2015). Stigma, sexual health, and human rights among women who have sex with women in Lesotho. Reproductive Health Matters, 23(46), 107-116. doi: 10.1016/j.rhm.2015.11.020

Rich, A. (2010). Heterossexualidade compulsória e existência lésbica. Bagoas, (5), 17-44. Recuperado de https://periodicos.ufrn.br/bagoas/article/vie w/2309

Rother, E. T. (2007). Revisão sistemática X revisão narrativa. Acta Paulista de Enfermagem, 20(2), 7-8. doi: 10.1590/S0103-21002007000200001

Schick, V., Pol, B. V. D., Dodge, B., Baldwin, A., \& Fortenberry, J. D. (2015). A mixed methods approach to assess the likelihood of testing for STI using self-collected samples among behaviourally bisexual women. Sex Transm Infect, 91, 329-333. doi: 10.1136/sextrans-2014-051842

Silberman, P., Buedo, P. E., \& Burgos, L. M. Barreras en la atención de la salud sexual en Argentina: percepción de las mujeres que tienen sexo con mujeres. Rev. salud pública, 18(1), 1-12. Recuperado de https://pesquisa.bvsalud.org/portal/resource /pt/mdl-28453149

UNAIDS - Programa Conjunto das Nações Unidas sobre HIV/AIDS. (2017). Guia de terminologia do UNAIDS. Brasília - DF. 


\section{Dados sobre os autores:}

- Amanda Dal Santo: Graduanda em psicologia na Universidade Estadual do Centro-Oeste (Unicentro), Campus Irati-PR.

- Gustavo Zambenedetti: Psicólogo (UFSM), Mestre e Doutor em Psicologia Social e Institucional (UFRGS). Professor do Departamento de Psicologia e do Programa de PósGraduação Interdisciplinar em Desenvolvimento Comunitário, Universidade Estadual do Centro-Oeste (Unicentro), Campus Irati-PR.

Declaração de Direito Autoral

A submissão de originais para este periódico implica na transferência, pelos autores, dos direitos de publicação impressa e digital. Os direitos autorais para os artigos publicados são do autor, com direitos do periódico sobre a primeira publicação. Os autores somente poderão utilizar os mesmos resultados em outras publicações indicando claramente este periódico como o meio da publicação original. Em virtude de sermos um periódico de acesso aberto, permite-se o uso gratuito dos artigos em aplicações educacionais e científicas desde que citada a fonte conforme a licença CC-BY da Creative Commons.

Creative Commons Atribuição 4.0 Internacional. 